\title{
An Intelligent Decision Support System for Managing Natural and Man-made Disasters
}

\section{Georgia Aifadopoulou}

Centre for Research and Technology Hellas - Hellenic Institute of Transport

$6^{\text {th }} \mathrm{km}$. Charilaou-Thermi Road, 57001, Thermi, Greece

E-mail: gea@certh.gr

\section{Emmanouil Chaniotakis}

Technical University of Munich, Arcisstraße 21, 80333, Munich, Germany

E-mail:m.chaniotakis@tum.de

\section{Iraklis Stamos}

IRU Projects, 71 Avenue de Cortenbergh, 1000 Brussels, Belgium

E-mail: iraklis.stamos@iruprojects.org

\section{Sokratis Mamarikas}

Centre for Research and Technology Hellas - Hellenic Institute of Transport

$6^{\text {th }} \mathrm{km}$. Charilaou-Thermi Road, 57001, Thermi, Greece

E-mail: smamar@certh.gr

\section{Evangelos Mitsakis}

Centre for Research and Technology Hellas - Hellenic Institute of Transport

$6^{\text {th }} \mathrm{km}$. Charilaou-Thermi Road, 57001, Thermi, Greece

E-mail: emit@certh.gr

Biographical notes: Georgia Aifadopoulou is Research Director at the Hellenic Institute of Transport. She holds a PhD in Transport and Logistics. Her research interests are related to logistics and the use of decision support systems in the transport domain.

Emmanouil Chaniotakis is a PhD candidate at the Technical University of Munich. He holds an MSc in Civil and Transportation Engineering. His research interests include optimization of transport systems and networks and transportation modeling.

Iraklis Stamos is Project Manager at IRU Projects. He holds an MSc in Transportation Engineering. His research interests include disaster management, planning transport networks for emergencies as well as logistics.

Sokratis Mamarikas is Research Associate at the Hellenic Institute of Transport. He holds an MSc in Mechanical Engineering. His research interests focus on environmental impacts of transport technologies as well as disaster and risk management.

Evangelos Mitsakis is Senior Researcher at the Hellenic Institute of Transport. He holds a PhD in Civil and Transportation Engineering. His research interests include Intelligent Transportation Systems as well as impact assessment of climate change on transport networks. 


\begin{abstract}
Climate change-induced disasters, in accordance with those related to human activities, are lately gaining in both severity and intensity. In view of their associated increased impacts on human assets and systems, public and private stakeholders have lately turned their attention to efficiently and effectively managing their effects, both prior to an event's occurrence, through better planning and protection, as well as during its temporal and spatial propagation at real-time through better operational adjustments and guidance to related authorities. In an effort to address this issue, the DECIDE project, funded by the IPA cross-border cooperation program, has developed, validated and tested under realistic conditions an intelligent Decision Support System (iDSS) for aiding public authorities in safe-proofing and protecting transport networks and operations in case of extreme weather events (EWE) and related natural hazards (NH). The iDSS is a webbased, GIS-enabled platform, able to suggest protection and management measures that optimally address occurring events, the affected modes of transport and transport networks and infrastructures. Optimal protection actions are derived from a database containing measures, strategies and policies that have been deployed at EU level in the last decades. In the framework of this paper, the iDSS architecture and main functional characteristics are presented, together with insights for its transferability to other locations.
\end{abstract}

Keywords: Disaster Management; Decision Support Systems; Open Source; GIS; Extreme Conditions Traffic Measures

\title{
1. Introduction
}

United Nations (UN) define disaster as "a sudden, calamitous event that causes serious disruption of the functioning of a community or a society involving widespread human, material, economic or environmental losses and impacts, which exceeds the ability of the affected community or society to cope using its own resources" (Soomaroo and Murray, 2012). Several types of events fall under this definition including both natural and manmade; fires, floods, landslides and industrial or technological accidents to name but a few. These disasters can induce severe impacts that may include among others loss of human life or injury, damage to properties and assets and social or economic disruption among others. This is also numerically reflected in the increasing occurrence and severity of such events. In order to mitigate and eliminate the potential impact of disasters, the resilience of systems needs to be improved (Mitsakis et al., 2014c; Stamos et al., 2015b). To that end, a field of research has emerged, namely Disaster Management (DM - also referred to as Emergency Management), that focuses on the development of methodologies, policies and practices serving this goal (Haddow et al., 2013). The UN has lately adopted the Disaster Risk Management concept in order to extend the general "risk management" term and refer to the strategies that focus on "avoiding, lessening or transferring adverse effects of hazards".

Disaster Management Research is classified into 4 main pillars: Prevention (or Mitigation), Preparedness, Response and Recovery (or reconstruction). Generally, it has been noted that the processes and decisions included in disaster management are highly complex (Haddow et al., 2013; Shan et al., 2012) and given the uniqueness of each disaster strike, limited opportunities are given in order to improve the process (Mendonça, 2007). For this reason, DSSs have been developed to assist authorities and respective stakeholders into taking optimal decisions that will reduce the exposure of people and systems to disasters' risks (Wallace and De Balogh, 1985).

DSSs can be characterized as computer based systems that aim at assisting decision-makers in the decisionmaking process. Essentially, DSSs help decision makers to take more productive, agile, innovative and reputable decisions (Holsapple, 2008). DSSs have lately received research attention as they can be used in several different cases, in which decisions might be difficult to take in an efficient and effective way, given their characteristics (i.e. complexity, dynamic character, agility) (Clemen and Reilly, 2013; Marakas, 2003). 
The main design goal is the understanding of the users' needs and the development of computer-based applications that can accommodate them (Fraunhofer, 2015). To this end, disaster management theory, models for rep-resenting disasters, theory of decision making and DSSs, knowledge acquisition and software development are investigated.

\section{Related Work}

\subsection{Disaster Management}

Serving the goal of mitigating and/or eliminating the impact of disasters to people and the environment, DM refers to the ongoing processes of preventing, mitigating, preparing, responding and reconstructing for and from a disaster. These processes, (referred to as "the disaster life cycle" or" comprehensive emergency management") are defined as circular rather than linear, to denote its ongoing and never ending character (Fagel, 2011). These processes form a comprehensive and integrated management framework that (a) addresses all disasters that might threaten a community, (b) is useful in all stages, (c) allows for seeks of resources and (d) knits together all partnerships (Fagel, 2011).

Specifically, Prevention (or mitigation) refers to preventative actions, which aim at mitigating or eliminating the potential effects of disasters. Mitigation plays a very important role in the disasters' management cycle, as it helps human communities to build resilience in order to mitigate or quickly absorb the effects of disasters, enhance local compliance with goals of the state and foster intergovernmental coordination (Lindell et al., 2007). Preparedness includes the planning of actions and measures that should be taken in case of a disaster. It is bounded with the choices and actions of the respective disaster managers for the area of their jurisdiction (Drabek, 1993; Lindell et al., 2001). Response refers to the period shortly after the occurrence of an adverse event. It refers to actions of temporally immediate nature, just after a hazard (Smith, 2011). It includes the implementation of the plans developed during the preparedness phase, as well as improvisation actions due to the unique character of each disaster (Lindell et al., 2007). Recovery (or reconstruction) aims at the restoration of the affected area to a normal state. This phase begins after the stabilization of the emergency and when there is no longer threat to property or life (Haddow et al., 2013). Disaster recovery copes with physical impacts (such as property damage) and social impacts (caused by human and property loss). It includes a series of actions that can take place in a sequence or simultaneously. The literature suggests that all phases should be structured in a sectorial differentiated way addressing various areas and aspects of the society, the economy and the environment (e.g. transport, water, energy) (Stamos et al., 2015a).

Disaster management is a complex procedure that requires continuous situational awareness. Disaster managers should be able to have the full picture of the situation that they are facing and also be prepared to act based on plans and structured improvisation. Communications and inter-organizational actions have been found to be of high importance as they can be crucial to the outcome of the disaster management. Furthermore, the decisions that disaster managers have to take are often subjective, which might lead to bad management is certain cases. Finally, the temporal dimension of a disaster could require actions to be taken in a very short time and many times simultaneously something that can lead to omissions.

\subsection{Decision Support Systems}

In the broad category of information systems, Managerial Support Systems (MSS) are used to support managerial activities. MSS include Decision Support Systems (DSS), Group Decision Support Systems (GDSS) and Expert Systems (ES) (Benbasat and Nault, 1990). DSS cover the spectrum of (mainly) computerbased systems that are developed to assist decision-makers in the decision-making process. Essentially, DSS help decision makers to take decisions that are more productive, agile, innovative and reputable (Holsapple, 
2008). Marakas (2003) asserts that the definition of DSS is a rather difficult task due to the existence of many applications and aggregates some common characteristics that characterize such a system (Marakas, 2003):

- Employed in semi-structured or unstructured decision context

- Intended to support rather than replace decision-makers

- Supports all phases of decision-making

- Focuses mainly on the effectiveness of decision-making process

- Is controlled by the DSS user

- Uses data and models

- Facilitates learning on the part of the decision-maker

- Is interactive and user friendly

- Is developed under iterative process

- Supports all managerial levels

- Is used for independent or interdependent decisions

- Supports individuals, groups or team based decision making context

DSS have been applied in a wide spectrum of applications, yet they are mainly found when decision making is hard and the decision context is rather complex (Clemen and Reilly, 2013; Marakas, 2003). Among others, DSS involve a decision context under the intention to support decisions without replacing decision-makers in all phases of the process using data and models in an interactive and user-friendly way, while facilitating learning on the part of the decision maker. DSS generally include different components that can be categorized in five systems (Marakas, 2003):

- Data Management System

- Model Management System

- Knowledge Engine

- User Interface

- User

The data management system includes all data related activities such as storage, retrieval and organization. The Model Management System includes the retrieval, storage, and management of the models included for quantitative or qualitative analysis required for decision making. The Knowledge Engine is responsible for the problem recognition and generation of solutions or any other function that is related to the problem solving process. It is the system that comprises data models in order to provide the user with what is required, in order to meet a decision using heuristics, rules, and any other logic required to help the decision maker. The user interface caters the interaction between the user and the other systems of the DSS mentioned earlier. Finally, the user of a DSS should always be taken into account as issues such as the related skills, motivations and knowledge domain have an important role in assisting on decisions. Those components constitute the basis of the architectural requirements that a Decision Support System must exhibit, as they cover all the important aspects that are comprised in a DSS system (Marakas, 2003).

One aspect of DSSs that is very important to take into account when designing a DSS is the decision process itself. First, it is important to understand why decisions are hard to be taken. In their work, Clemen and Reilly (2013) define the context in which some decisions are rather hard to take, by identifying 4 key sources of difficulty: Complexity, Uncertainty, Multi-Objectiveness and Multi-Perspectives. Simon (1960) proposed a three phase process model describing the problem solving process. First, the Intelligence phase is introduced, where the decision maker searches/scans for information suggesting the presence of a problem that he owns, with ownership to be defined as the ability to have a solution. The identification of a problem and its definition triggers activities related to the analysis of the problem, the definition of the problem solving strategy and the formation of possible alternatives (Design phase). Finally the Choice phase, is the phase during which the 
choice is made given the problem and the objectives that the decision maker has set. In addition, Marakas (2003) addressed the subjects of structuredness of decision, cognitive limitations and perception to be important when studying why decisions are hard to take. These factors actually shape the decision environment. Perception is a special kind of cognitive limitation (Marakas, 2003) that can limit the decisionmaking process. It can be understood as a filter based on several factors such as experience, personal goals, beliefs and values (Marakas, 2003).

DSSs constitute a collection of research fields, which are basically aiming at understanding and providing the context for better decision making. Several aspects should be taken into account when designing such a system with the decision making process and the decision maker characteristics to play a central role. Furthermore, the concept of knowledge is of high importance as it allows decision makers to make decisions based on facts and limit the effects of cognitive limitations and perception. Finally, DSS cases presented provide a basis of identification of capabilities of DSS and its applications.

\subsection{Decision Support Systems for Disaster Management}

A wide spectrum of decisions in the context of DM fit the criteria suggested by Marakas (2003) and Clemen and Reilly (2013) on hard decisions. Decision makers are often exposed to a situation which is complex with a high degree of uncertainty due to changing characteristics of disasters (i.e. magnitude, propagation), governed by multi-objectiveness that can be described from multiple perspectives (Fagel, 2011). Furthermore, disasters are events that occur scarcely and require immediate actions (Baker, 1991); as such, decision makers are bounded by their limited experience, cognitive limitations and perception (Marakas, 2003). Given the above mentioned, DM can be consider ideal, for adopting DSS that would allow for better decisions to be taken.

\section{Aspects of Theoretical Foundations for DSS for DM}

One of the first attempts to draft the design of DSS for DM was presented by Wallace and De Balogh (1985). In their work, Wallace and De Balogh (1985) defined the context in which a DSS could improve all 4 stages of disaster management by indicating which components of DSS systems and technologies available at that period could be used. Furthermore, they defined decisions to be taken based on their structuredness in order to prioritize for the development of DSS. From the publication of that paper, several other papers were published on the way that DSSs can improve DM, which have identified some key aspects of those systems.

To begin with, an important aspect is the implementation of actions based on accurate situational awareness in all phases of DM (Horita and De Albuquerque, 2013). During the mitigation and preparedness phase, situational awareness refers to the knowledge concerning exposure, vulnerability, level of preparedness and mitigation, as well as availability of resources (Lindell et al., 2007, 2001; Paton and Johnston, 2001; Schneider and Schauer, 2006; Weichselgartner, 2001). During disaster response and reconstruction, a vast amount of literature concerning Disaster Management suggests that the decision maker should have accurate and welltimed information about the situation in hand (Fagel, 2011; Haddow et al., 2013; Lindell et al., 2007), in order to evaluate and act. It should be noted, that this information, does not necessarily prescribe a set of "correct" actions to be taken and that decision makers have to understand the impact of their decision (Levy et al., 2005).

In this context, situational awareness is enhanced by including indications of what would be the results of complex actions by either real-time evaluation or scenario analysis, based on analytical models or simulations of the situation examined (Wu et al., 2008). Situational awareness allows for reducing the effects of decision makers' cognitive limitations and perception to the decision process (Marakas, 2003), which is found to be increased during high stress situations (Van de Walle and Turoff, 2008).

Another key aspect of DM commonly discussed as a negative issue is that decision makers have limited experience in handling disasters due to the (relative) scarcity of such events. Limited experience can be rather risky as under high stress and anxiety, situations individuals tend to rely more on internal hypothesis testing 
and well-learnt responses (Staw et al., 1981). Furthermore, Van de Walle \& Turoff (2008) pointed out that integration and collaboration among different entities require trust and understanding which is only built when collaborating even in terms of exercises.

Training could be in part made possible with the use of a DSS adopting scenarios, which employ models and simulations to understand and allow for evaluating impact of any action. Furthermore, it is widely pointed out that inter-organizational training should be taken into account as it is a rather common case during DM (Mendonça, 2007).

Central for DM is also the provision of reliable, fast and effective actions enhanced by effective communications, which emerge as an issue from the past experiences of problematic response due to miscommunications (Rolland et al., 2010). For example, in many disasters cases, the resources allocation (human, machinery, money etcetera) is handled by different organizations, which are required to communicate during allocation. However, due to miscommunications, there are cases of over- and under-demand provision of resources (Meissner et al., 2002; Rolland et al., 2010). Effectiveness of communications is enhanced by clear structures and communication channels. DSS for DM are ideal for providing such environment in which effective actions and communications can be achieved using standardised communication protocols (Rieser et al., 2015). Improvisation is considered to be a vital part of DM for two reasons: a) it allows for flexibility in handling unexpected phenomena and $b$ ) it is impossible to examine all the scenarios possible during preparedness (Mendonça, 2007; Kreps, 1991). Mendonça et al. (2001) suggest that there are two stages in emergency improvisation: first, there is the recognition that there is no plan for the situation, and second, there is the decision to take a decision based on improvisation. DSS for DM structure give the opportunity to enhance situational awareness during the recognition phase and also provides room for evaluation of the improvisation results.

\section{4. iDSS Functionalities}

The functionalities of the iDSS have been based on a user needs survey conducted within the framework of the DECIDE project (Chrysostomou \& Mitsakis, 2015). The main user needs identified include:

- Improvement of the communication and the common understanding between stakeholders

- Facilitation of the early warning and notification

- Direct access on a number of data displayed on an electronic map, related to disasters and the overall disaster management

- Enhancement of the planning process, through indication of measures, processes and management of resources

- Increase of the level of preparedness, through the provision of access on information regarding weather forecasting and expected hazards

- Improvement of the assessment of the current situation in the first phases of a disaster

- Improvement of the response, through rapid mapping capabilities - Improvement of the operational capacity of the response in disasters providing routing indications, resources' allocation advices and traffic management indications.

The functions of the iDSS arise from the user needs that have been recorded within the DECIDE project (Chrysostomou \& Mitsakis, 2015) and are applicable in all the phases of the disaster management cycle.

A very important but on the same time rather time consuming aspect of DM is the organization and communication of the related authorities and the people that take part in the actual disaster management. The iDSS facilitates a spectrum of functions that allow for quick communication of users using Short Message Service (SMS), automated e-mail service and alerts to users of the system. This functionality in conjunction to the fact that all authorities have access to the same database for spatial information that describe a hazard allow for a reduction of the communication overhead and the explanation of the situation in hand. 
A key functionally of the DECIDE's iDSS is the resource and personnel specification. Its authority has the ability to insert, view, modify and use for analysis the resources under the jurisdiction of the authority. This functionality allows for enhanced situational awareness, communications, and for future facilitation of administrative actions.

GIS Data Viewers allow for the representation of important spatial information in DM. Such information can be previous disasters information, resources, boundaries of jurisdiction, important infrastructures and information that the user can consider as important. Viewers allow for the insertion of data considered as important by users of the iDSS.

Legislation viewers and search allow for the insertion, management and view of the Disaster related legislation and other pertinent documents that govern Disaster Management. This functionality provides a powerful tool that gives quick access to the procedures to be followed and actions to be taken during DM.

The protection measures functionality of the iDSS allows for the insertion, management and plan of the protection measures that should be taken during the mitigation and preparedness phase of DM. This functionality allows for tabular or timeline view of commonly implemented protection measures per hazard and examined area, assisting decision makers to implement them based on multi-objective decision making.

Another need identified by the user needs study was the allocation of the civil protection units based on allocation algorithms and given constraints that should be met (Mitsakis et al., 2014b). The iDSS includes this functionality and allows for allocation of the units that each authority has under their jurisdiction based on the principle of maximum coverage (or smallest distance).

In most cases of applied response in DM, routing under emergency conditions takes place on empirical awareness of the transportation network state. This practice could however be problematic during DM as the situation in hand is most times different to everyday traffic conditions. For this reason the routing under emergency conditions functionality is provided that would allow in future versions the incorporation of realtime traffic conditions and traffic demand prediction in the estimation of the shortest path for emergency vehicles.

In most cases of applied response in DM, traffic management is based (as emergency routing) on empirical awareness of the situation in hand and in former practice. Again, as its case of disaster is unique, the traffic management measures that should be taken should be optimized based on information that corresponds to the actual situation and more often than not, address evacuation perspectives (Ayfadopoulou et al., 2012; Mitsakis et al., 2014a). This need is covered by the introduction of the traffic management functionality that cover a spectrum of actions to be taken in order to increase the performance of the transportation system during the phase of response in the DM process.

An important user need identified from the questionnaires and the review of the state-of-the-art on DSS for DM was the geo-referenced reporting of events. The users are allowed to upload a picture of the event and include the hazard and the location.

Based on this analysis, the design of the iDSS includes the following functionalities:

- Communications and alerts

- Resources and personnel specification

- GIS Viewers

- Legislation Viewers and Search

- Protection measures (Planning)

- Allocation of civil protection units (Planning)

- Routing under emergency conditions

- Traffic management functions

- Event Reporting

Further to the above functionalities, the iDSS has an inherent user management functionality, fit to the needs of civil protection related authorities, the details of which are presented in (Mitsakis et al., 2016) 


\section{5. iDSS Architecture}

The iDSS Architecture is based on the architectural components suggested by Marakas (2003) and on the user needs that shape the iDSS functionalities. An abstract schematic representation of the architecture able to cater for the DM needs and to incorporate the components above is presented in the Figure 1. It should be noted that given the open source character objective, all the tools and libraries included in the systems' architecture are characterised as open source or open in case of data.

\subsection{Architectural Components}

The Data Management System incorporates all the data related tools and caters for the data related processes. Efficient storage of data is a core function of each web application design, while depending on the required data model, different database systems can be used. The main data model requirements of the DECIDE project software clearly indicate the need of storing a vast amount of georeferenced data (coordinates and related information) as well as capabilities for storing other data types. A database system that caters the software requirements is PostGIS, which is an open source spatial database based on PostgreSQL (www.postgresql.org). A database subsystem is required for document management. Its design requires the incorporation of Content Management System (CMS) and also document indexing, search and tagging system. The document management requirement is facilitated by the open source version of Alfresco (www.alfresco.com).

The Model Management System incorporate all mathematical models to describe phenomena such as hazard propagation and analysis tools to allow for estimation of indicators such network criticality, shortest path identification, solutions to Facility Allocation Problem and others. All the above mentioned are coded in JAVA runtime in modelling or analysis classes environment. In most cases open source libraries are used for the incorporation of the required functionalities (see Section 4). Given the transport oriented initial development of the software, the Open Source Routing Machine (OSRM) (www.project-osrm.org) and JAVA Optimization libraries (such as JOptimizer - www.joptimizer.com) are employed.

The knowledge engine is the link that connects the Data Management System, the Model Management System and the User Interface. In a sense it is the framework that cater for the definition of the process flows, the rules that apply

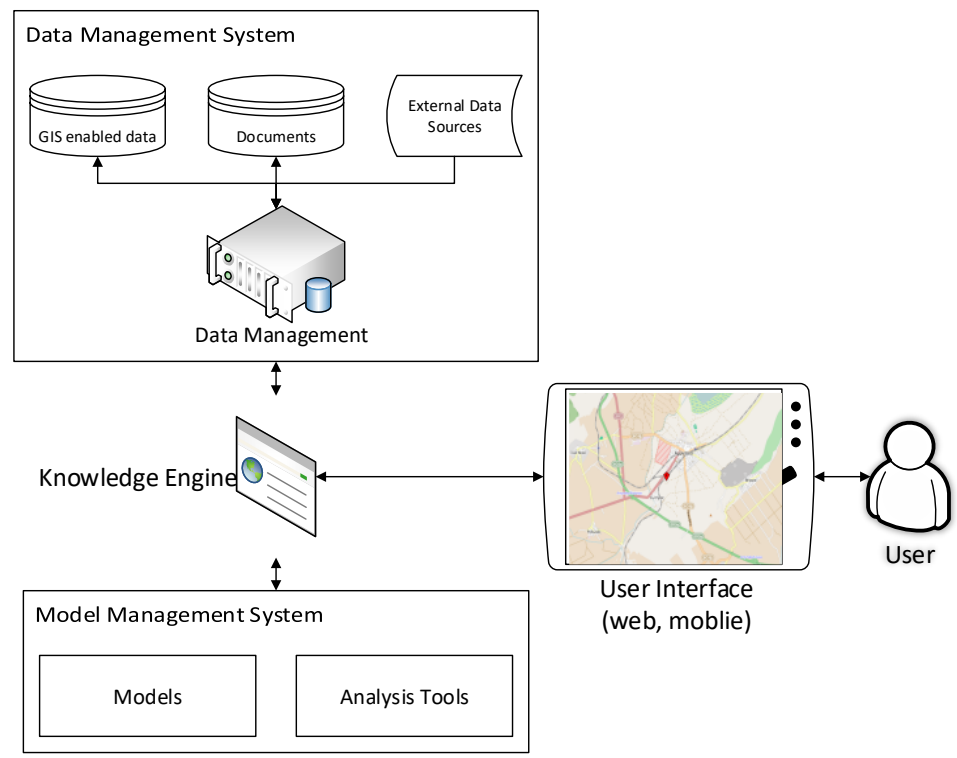

Fig. 1. Abstract Schematic Representation of the iDSS Architecture 
and the actual interaction between the user (via the user interface) and the Data Management System/ Model Management System. Within the iDSS architecture, the knowledge engine is used, in order to:

- facilitate the keyword and tag search

- facilitate the Measures component, providing features that can extract knowledge through filters

- extract suggestions on facilities allocation in the case of an emergency

The Play Framework (www.playframework.com) has been selected, since it is one of the most modern web \& mobile application frameworks. Play is open-source and is also built on the Model-View-Controller (MVC) pattern (Krasner and Pope, 1988).

The user interface is designed with focus on what users might need to do and ensures that it has elements that are easy to access, understand and use.

Some of the basic practices that we have followed while designing this application are:

- $\quad$ Keep the interface simple. That means to try to include only the necessary components and be clear in the interface's language.

- Be purposeful in page layout. It is important to structure the page based on importance. Careful placement of items can draw attention to the most important pieces of information and make the whole interface more readable.

- Use common UI elements. Such elements can be checkboxes, dropdown lists, buttons, pagination, notifications, modal windows etc. By using common elements, users feel comfortable and are able to get things done faster.

- $\quad$ Use typography to create hierarchy and clarity. Different sizes, fonts and arrangement of the text can help increase legibility and readability.

- One primary action per screen. Every screen we design should support a single action of real value to the person using it. This reduces confusion and makes the interface easier to learn.

The entities specified as users of the iDSS system are authorities such as the General Secretariat at the central level and the Decentralized Administrations, the Regions and the Municipalities at a more local level have been defined as beneficiaries' bodies. The system should also be addressed to operational bodies such as the Police and the Fire Brigade, enhancing the operational efficiency of their forces in disaster's response, while the users can specify the information allowed to be shared with other entities. The identification of those users was based on a review of the civil protection legislation in Greece. Each (physical) user registered to the system can be allocated to one of the entities. The users of the iDSS can do specific operations based on which group they belong to. Moreover, more than one administrator can be defined for the smooth operation of the system. The administrator is responsible for the maintenance of the system, the installation of new instances and the monitoring of the network communication. The administrator can perform all the operations provided by the application.

The system of iDSS is built around a Java framework (Play Framework) upon which connect all the other components like GeoServer, Alfresco, OpenLayers, OSRM and a PostGIS database. The selected framework is designed over the MVC pattern which was described earlier in the Suggested Architecture; the detailed schematic representation of which is presented in Figure 2. 


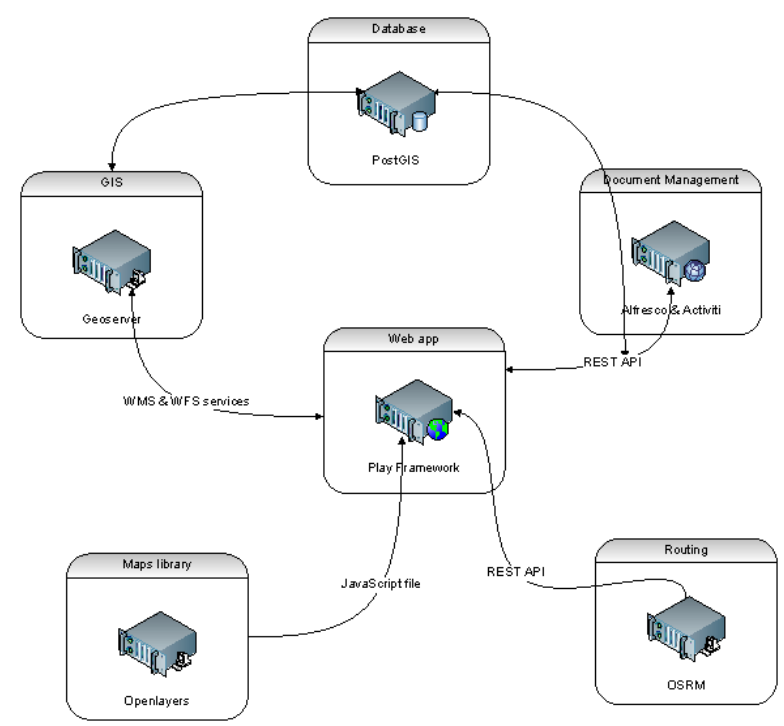

Fig. 2. Detailed Schematic Representation of the iDSS Architecture

\subsection{System Transferability and Extendibility}

The proposed architecture and the included components were depicted such that they would allow for easy transferability to different areas. This is supported on the fact that the main data sources used are well maintained world databases (such as the Openstreetmap database) and the design of the algorithms allows for direct transferability given same field names. Finally, users are able to alter the organizations and entities using the software interface based on the country's DM system and to upload DM-related documents and data without any restriction.

In the same level, the open source character of all the components of the architecture allow for the development of extensions suitable for each user's group. This applies also on the development of models that can also be used for testing various solutions on scenario planning level.

\section{Conclusions}

This paper presents the architecture and the implementation of a Decision Support System for Disaster Management (iDSS), developed in the context of the DECIDE project. The added value of the DECIDE project's iDSS is the acknowledgement of civil protection activities by underlining its vital role in protecting the environment, human lives and critical infrastructures.

The findings of the literature review and the user needs defined allowed for the specification of the required functionalities. The architecture is based upon the premises of the generic DSS architecture model, while the detailed architecture incorporates the tools considered to be suitable for the implementation based on recorded user needs.

The inclusion of a decision support system in civil protection processes implies the recognition of the roles of actors in the planning process, the realization of broader needs, the recognition and study of best practices and corresponding systems, the identification of bodies' roles and the final system design based on synergies and processes developed in disaster management. Using a common integrated GIS-based system by all involved bodies at different levels of administration is a necessity in order to meet various civil protection and disaster management requirements, as it allows the easy exchange of geospatial data between involved actors, the provision of early warning in case of emergencies and disasters and the effective dissemination of information related to catastrophic events. As necessary steps to the further development of the existing system are the collection and digitalization of various kinds and volumes of geospatial data, which requires intensive 
effort, and their integration into existing operations with a view of jointly processing and managing such data by all involved actors. Another important parameter is the harmonization of the system with historical disaster databases officially used at European level, as well as the collaboration with early warning systems.

\section{Future Research Directions}

Upon the topic of this paper, some future research is needed for the comparison of the findings of the described methodology, as designed within the framework of the DECIDE project, with the findings of international research in order to investigate if the structure and functionalities of the DSS apply for cases of cross border incidents. Another issue which needs to be investigated is the factors which could limit the use of the DSS. Factors such as legislation concerning bilateral cooperation, language barriers and absence of Standard Operating Procedures for cross border events can affect the functionality of the DSS.

\section{References}

Ayfadopoulou, G., Stamos, I., Mitsakis, E., Grau, J.M.S., 2012. Dynamic Traffic Assignment Based Evacuation Planning for CBD Areas. Procedia - Soc. Behav. Sci. 48, 1078-1087. doi:http://dx.doi.org/10.1016/j.sbspro.2012.06.1084

Baker, E.J., 1991. Hurricane evacuation behavior. Int. J. Mass Emerg. Disasters 9, 287-310.

Benbasat, I., Nault, B.R., 1990. An evaluation of empirical research in managerial support systems. Decis. Support Syst. 6, $203-226$. doi:http://dx.doi.org/10.1016/0167-9236(90)90015-J

Chrysostomou, K., Mitsakis, E., 2015. DECIDE Project Deliverable 3.4.1 "Integration of the iDSS in regional policy level"

Clemen, R., Reilly, T., 2013. Making hard decisions with DecisionTools. Cengage Learning.

Drabek, T.E., 1993. Major themes in disaster preparedness and response research, in: Major Themes in Disaster Preparedness and Response Research. Research Seminar on Socioeconomic Aspects of Disaster in Central America.

Fagel, M.J., 2011. Principles of Emergency Management: Hazard Specific Issues and Mitigation Strategies. CRC Press.

Fraunhofer, 2015. D8.5 Final Report IDIRA Project. Dresden.

Haddow, G., Bullock, J., Coppola, D.P., 2013. Introduction to emergency management. Butterworth-Heinemann.

Holsapple, C.W., 2008. Decisions and knowledge, in: Handbook on Decision Support Systems 1. Springer, pp. 21-53.

Horita, F.E.A., De Albuquerque, J.P., 2013. An approach to support decision-making in disaster management based on volunteer geographic information (VGI) and spatial decision support systems (SDSS), in: Proceedings of the 10th International Conference on Information Systems for Crisis Response and Management, Baden-Baden, Germany.

Krasner, G.E., Pope, S.T., 1988. A description of the model-view-controller user interface paradigm in the smalltalk- 80 system. J. object oriented Program. 1, 26-49.

Kreps, G.A., 1991. Organizing for emergency management. Emerg. Manag. Princ. Pract. local Gov. 30-54.

Levy, J.K., Gopalakrishnan, C., Lin, Z., 2005. Advances in Decision Support Systems for Flood Disaster Management: Challenges and Opportunities. Int. J. Water Resour. Dev. 21, 593-612. doi:10.1080/07900620500258117

Lindell, M.K., Prater, C., Perry, R.W., 2007. Introduction to emergency management. WILEY, Hoboken, NJ.

Lindell, M.K., Tierney, K.J., Perry, R.W., 2001. Facing the Unexpected:: Disaster Preparedness and Response in the United States. Joseph Henry Press.

Marakas, G.M., 2003. Decision support systems in the 21st century. Prentice Hall Upper Saddle River, NJ.

Meissner, A., Luckenbach, T., Risse, T., Kirste, T., Kirchner, H., 2002. Design challenges for an integrated disaster management communication and information system, in: The First IEEE Workshop on Disaster Recovery Networks (DIREN 2002). 
Mendonça, D., 2007. Decision support for improvisation in response to extreme events: Learning from the response to the 2001 World Trade Center attack. Decis. Support Syst. 43, 952-967. doi:10.1016/j.dss.2005.05.025

Mendonça, D., Beroggi, G.E.G., Wallace, W.A., 2001. Decision support for improvisation during emergency response operations. Int. J. Emerg. Manag. 1, 30-38.

Mitsakis, E., Stamos, I., Aifadopoulou, G., Mamarikas, S., 2016. DECIDE Project Deliverable 2.1.1 "Decision Support System for Disaster Emergency Management"

Mitsakis, E., Stamos, I., Diakakis, M., Salanova Grau, J.M., 2014a. Impacts of high-intensity storms on urban transportation: applying traffic flow control methodologies for quantifying the effects. Int. J. Environ. Sci. Technol. 11, 2145-2154. doi:10.1007/s13762014-0573-4

Mitsakis, E., Stamos, I., Grau, J.M.S., Aifadopoulou, G., 2014b. Optimal allocation of emergency response services for managing disastersnull. Disaster Prev. Manag. An Int. J. 23, 329-342. doi:10.1108/DPM-10-2013-0182

Mitsakis, E., Stamos, I., Papanikolaou, A., Aifadopoulou, G., Kontoes, H., 2014c. Assessment of extreme weather events on transport networks: case study of the 2007 wildfires in Peloponnesus. Nat. Hazards 72, 87-107. doi:10.1007/s11069-013-0896-3

Paton, D., Johnston, D., 2001. Disasters and communities: vulnerability, resilience and preparedness. Disaster Prev. Manag. An Int. J. 10, 270-277. doi:10.1108/EUM0000000005930

Rieser, H., Dorfinger, P., Nomikos, V., Papataxiarhis, V., 2015. Sensor Interoperability for Disaster Management, in: Sensors Applications Symposium 2015.

Rolland, E., Patterson, R.A., Ward, K., Dodin, B., 2010. Decision support for disaster management. Oper. Manag. Res. 3, 68-79.

Schneider, P.J., Schauer, B.A., 2006. HAZUS—its development and its future. Nat. Hazards Rev. 7, 40-44.

Shan, S., Wang, L., Li, L., 2012. Modeling of emergency response decision-making process using stochastic Petri net: An e-service perspective. Inf. Technol. Manag. 13, 363-376. doi:10.1007/s10799-012-0128-7

Simon, H.A., 1960. The new science of management decision.

Smith, S.L., 2011. An Analysis of Authority Issues within National Response Framework ESF \#6 - Mass Care, Housing and Human Services Policy. Risk, Hazards Cris. Public Policy 2, 1-14. doi:10.2202/1944-4079.1071

Soomaroo, L., Murray, V., 2012. Disasters at mass gatherings: lessons from history. PLoS Curr. 4.

Stamos, I., Mitsakis, E., Salanova Grau, J.M., 2015a. Roadmaps for Adaptation Measures of Transportation to Climate Change. Transp. Res. Rec. J. Transp. Res. Board 2532, 1-12. doi:10.3141/2532-01

Stamos, I., Mitsakis, E., Salanova, J.M., Aifadopoulou, G., 2015b. Impact assessment of extreme weather events on transport networks: A data-driven approach. Transp. Res. Part D Transp. Environ. 34, 168-178. doi:http://dx.doi.org/10.1016/j.trd.2014.11.002

Staw, B.M., Sandelands, L.E., Dutton, J.E., 1981. Threat Rigidity Effects in Organizational Behavior: A Multilevel Analysis. Adm. Sci. Q. 26, 501-524. doi:10.2307/2392337

Van de Walle, B., Turoff, M., 2008. Decision support for emergency situations. Inf. Syst. E-bus. Manag. 6, $295-316$. doi:10.1007/s10257-008-0087-z

Wallace, W.A., De Balogh, F., 1985. Decision support systems for disaster management. Public Adm. Rev. $134-146$.

Weichselgartner, J., 2001. Disaster mitigation: the concept of vulnerability revisited. Disaster Prev. Manag. 10, 85-95. doi:10.1108/09653560110388609

Wu, S., Shuman, L., Bidanda, B., Prokopyev, O., Kelley, M., Sochats, K., Balaban, C., 2008. Simulation-based Decision Support System for Real-time Disaster Response Management, in: Proceedings of the 2008 Industrial Engineering Research Conference. pp. 58-63. 